\title{
Screening Value of Timed Up and Go Test for Frailty and Low Physical Performance in Korean Older Population: The Korean Frailty and Aging Cohort Study (KFACS)
}

\author{
Hee-Won Jung ${ }^{1, *}$, Sunyoung Kim ${ }^{2,}$, Il-Young Jang ${ }^{1}$, Dong Wook Shin ${ }^{3}$, Ji Eun Lee ${ }^{4}$, Chang Won Won ${ }^{5}$ \\ ${ }^{1}$ Division of Geriatrics, Department of Internal Medicine, Asan Medical Center, Seoul, Korea \\ ${ }^{2}$ Department of Family Medicine, College of Medicine, Kyung Hee University, Seoul, Korea \\ ${ }^{3}$ Department of Family Medicine, Samsung Medical Center, Sungkyunkwan University School of Medicine, Seoul, Korea \\ ${ }^{4}$ Department of Family Medicine, CHA Bundang Medical Center, CHA University, Seongnam, Korea \\ ${ }^{5}$ Elderly Frailty Research Center, Department of Family Medicine, College of Medicine, Kyung Hee University, Seoul, Korea
}

Corresponding Author:

Chang Won Won, $\mathrm{MD}, \mathrm{PhD}$

Elderly Frailty Research Center,

Department of Family Medicine,

College of Medicine, Kyung Hee

University, Kyungheedaero 23,

Dongdaemun-gu, Seoul 02447, Korea

E-mail: chunwon@khmc.or.kr

ORCID:

https://orcid.org/0000-0002-6401-2735

*These authors contributed equally to this work.

Received: October 13, 2020

Revised: November 19, 2020

Accepted: November 21, 2020
Background: We evaluated the validity of the Timed Up and Go test (TUG) to screen for physical frailty and low physical performance in a nationwide community-dwelling Korean older population. Methods: We used baseline records of 3,010 ambulatory participants with TUG data from the Korean Frailty Aging Cohort Study from 2016 to 2017. The population-specific distribution of TUG was assessed. Physical frailty was defined as $\geq 3$ positive items in the 5-item Cardiovascular Health Study (CHS) frailty scale, and low physical performance was assessed as Short Physical Performance Battery (SPPB) scores $\leq 9$ (ranging from 0 to 12). Results: In men $(n=1,429)$ and women $(n=1,581)$, the mean TUG times were $10.3 \pm 2.7$ seconds and $10.2 \pm 3.0$ seconds, respectively. The cut-off TUG times for the worst quintile were 11.8 seconds in men and 12.5 seconds in women. The TUG time was correlated with both the CHS frailty scale score (standardized beta $[B]=0.36, p<0.001)$ and SPPB total score $(B=-0.22, p<0.001)$ in the linear regression analysis adjusted for age and sex. In the receiver operating characteristic analysis, the performance of TUG in identifying physical frailty, calculated as the area under the curve (AUC), was 0.87 , while the AUC of TUG in identifying low physical performance according to SPPB was 0.86. Conclusion: In the Korean older population, TUG can be a simple measure to identify physical frailty and low physical performance so as to identify populations that may benefit from in-depth geriatric assessments.

Key Words: Frailty, Diagnosis, Screening, Korea, Physical performance

\section{INTRODUCTION}

Frailty, a state of increased vulnerability to possible stressors with decreased physiological reserve, is a common geriatric problem and is associated with adverse health outcomes in older adults. ${ }^{1,2)}$ To define and assess frailty, various concepts and tools have been developed and validated across populations. ${ }^{2,3)}$ Among these concepts and tools, the frailty phenotype to capture physical changes associated with human aging is a widely accepted way to delineate the frailty spectrum. ${ }^{4)}$ Specifically, the frailty phenotype criteria of the Cardiovascular Health Study (CHS) and the Short Physical Performance Battery (SPPB) are commonly used in studies to identify people with frailty. ${ }^{4,5)}$

The Timed Up and Go test (TUG), which measures the time needed to get up from a chair, walk $3 \mathrm{~m}$, and then return and sit back on a chair, is widely used as a simple screening tool to assess physical frailty in older adults. ${ }^{6,7)}$ Previous studies have demonstrated the utility of TUG in identifying the frailty phenotype in 
western populations. ${ }^{8,9)}$ Since the test includes fragments of movements included in SPPB, such as chair rise and walking, TUG can also be used as a quick measure of physical performance. Expectably, reports have shown the outcome relevance of TUG, including its association with the future incidence of functional decline, fracture, heart diseases, and Parkinsonism., ${ }^{710-13)}$

However, the relationship between TUG time and physical frailty or physical performance has been less studied in the Korean population. Therefore, we evaluated the associations between these measures and assessed the validity of TUG as a screening tool for frailty phenotype defined by the CHS criteria and low physical performance determined by SPPB in a nationwide community-dwelling Korean older population.

\section{MATERIALS AND METHODS}

\section{Study Population and Protocol}

This study used baseline records of 3,010 ambulatory participants who were aged 70-84 years, had geriatric assessments and TUG data that were assessed from 2016 to 2017 in the Korean Frailty Aging Cohort Study (KFACS), a nationwide multicenter longitudinal study conducted in 10 urban, rural, and suburban communities across Korea. Detailed descriptions of the KFACS design and measures are published elsewhere. ${ }^{14)}$ Briefly, the participants were recruited based on age- and sex-specific strata, and residents with no plans to move out during the following 2 years and with no difficulties in conversing were eligible to participate in this study. People with uncontrolled hypertension ( $>180 / 100 \mathrm{mmHg}$ ), cerebrovascular accident or myocardial infarction within the past 6 months, or active malignancy currently under treatment were excluded. The study protocol was reviewed and approved by the Institutional Review Board of Kyung Hee University Hospital (No. 2020-09-049) and complied with the ethical rules for human experimentation described in the Declaration of Helsinki. Informed consent was obtained from all participants or their proxy.

\section{Physical Performance Assessments}

For TUG, time was measured as the time required for the participants to rise from a straight-backed chair at once, walk at a comfortable pace for $3 \mathrm{~m}$, turn around and walk back to the chair, and sit down. ${ }^{6)}$ The participants were instructed to start the TUG maneuver immediately after hearing the "Start" command. The participants were allowed to use walking aids (e.g., cane or walker) during TUG. The usual gait speed over a distance of $4 \mathrm{~m}$ was measured using an automatic gait speed meter (Gaitspeedometer, Dyphi, Daejeon, Korea), with acceleration and deceleration phases of $1.5 \mathrm{~m}$ each. ${ }^{15)}$ The participants were asked to perform TUG by walking at their usual pace. The participants performed the test two times, and the results were averaged. The five-time-sit-tostand test measured the time required to stand five times from a sitting position from a straight-backed chair as quickly as possible without using the arms. We also used SPPB, which included three components - standing balance, walking speed, and chair rise test- to assess physical performance according to recommendations from previous studies. ${ }^{15)}$ Each item of SPPB was scored based on a 0 to 4 point scale, with the total score ranging from 0 (worst) to 12 (best) points. According to literature, low physical performance was defined as an SPPB total score $\leq 9 .{ }^{16)}$ SPPB parameters were available for 1,429 men and 1,581 women.

\section{CHS Frailty Phenotype Scale}

To assess physical frailty, we used the CHS scale, which comprises five components, namely unintended weight loss, poor grip strength, exhaustion, reduced walking speed, and low physical activity level. ${ }^{4)}$ We defined unintended weight loss as $\geq 4.5 \mathrm{~kg}$ in the previous year. Poor grip strength was defined as the lower 20th percentile of grip strength (maximal grip strength in kg after measuring twice for each hand using a hand grip dynamometer [T.K.K.5401; Takei Scientific Instruments Co., Tokyo, Japan]), stratified by sex and body mass index (BMI) quartiles based on the KFACS baseline survey. ${ }^{17)}$

To quantify exhaustion, we used the following statements from the Center for Epidemiological Studies-Depression (CES-D) scale for 3 or more days in a week: "I felt that everything I did was an effort" or "I could not get going" ${ }^{18)}$ For slowness, we used the lowest $20 \%$ of a 4-m gait speed stratified by sex and height based on KFACS data. ${ }^{17)}$ Low physical activity level was defined as an energy expenditure level $494.6 \mathrm{kcal}$ per week for men and below 283.5 kcal per week for women according to the International Physical Activity Questionnaire. These values corresponded to the lowest $20 \%$ of the sex-specific total energy consumed according to a general population-based survey of older adults. ${ }^{17)}$ Participants positive for three or more of these items were classified as frail; those positive for 1-2 items were classified as prefrail, and those with no positive items for any of the criteria were classified as robust. The CHS frailty parameters were available for 1,382 men and 1,523 women.

\section{Other Measurements}

Information on participant age, marital status, education level, drinking status, smoking status, comorbidities, and functional capacities was collected during face-to-face interviews. Alcohol consumption was defined as three or more alcoholic drinks per week, while smoking was defined as a lifetime consumption of 100 or 
more cigarettes. To assess functional capacities, Korean Mini-Mental State Examination, ${ }^{19)}$ Geriatric Depression Scale Short Form, ${ }^{20}$ ) and minimal nutritional assessment questionnaires ${ }^{21)}$ were used. BMI was calculated as weight divided by the square of the height $\left(\mathrm{kg} / \mathrm{m}^{2}\right)$.

\section{Statistical Analysis}

Continuous variables are shown as mean \pm standard deviation, while categorical variables are described as number and percentage. T-tests were used for continuous variables and $\chi^{2}$ tests for categorical variables. Skewness was calculated, and histograms were used to display the distribution of TUG time in men and women. The commonly used percentile values for epidemiological studies were also calculated. We used linear regression analysis to assess the correlations between TUG time, total number of positive items according to the CHS criteria, and SPPB total score. Linear regression analyses were also used to assess the correlations between TUG time, item specific and total CHS frailty scale and SPPB scores. Receiver operating characteristic (ROC) analyses were performed to evaluate the classification performance of TUG for frailty assessment using the CHS criteria and low physical performance; the sensitivities and specificities for various TUG times ( $\geq 8$ to $\geq 16$ seconds) to classify frailty and low physical performance were also calculated. Statistical analyses were performed using Stata 15.0 (Stata Corp, College Station, TX, USA), with statistical significance set at a two-sided p-value $<0.05$.

\section{RESULTS}

\section{General Characteristics and Distributions of TUG Time}

The mean age of the 3,010 participants was $76.5 \pm 3.9$ years, and 1,429 (47.5\%) participants were men. The baseline demographic, anthropometric, and functional characteristics are shown in Table 1. The mean TUG times were $10.3 \pm 2.7$ seconds in men and $10.8 \pm 3.0$ seconds in women. The TUG time was significantly higher in women than in men $(p<0.001)$. The histogram in Fig. 1 displays the distribution of TUG times in men and women, with commonly used percentile cut-offs of TUG time in the study population. Specifically, the TUG cut-off times for the worst quintile was 11.8 seconds in men and 12.5 seconds in women. Assessment showed that TUG was right skewed in both men and women $(\mathrm{p}<0.001)$.

\section{Correlations between TUG Time and Physical Performance and Frailty Status}

TUG time was positively associated with age (standardized beta $[\mathrm{B}]=0.38, \mathrm{p}<0.001)$ and CHS frailty scale score $(\mathrm{B}=0.24$, $\mathrm{p}<0.001)$ and negatively associated with SPPB total score $(\mathrm{B}=-$ $0.36, \mathrm{p}<0.001$ ) in the linear regression analysis (Supplementary Fig. S1). The correlation between TUG time and CHS frailty scale score remained significant $(B=0.22, \mathrm{p}<0.001)$ after adjusting for age and sex. Similarly, the correlation between TUG time and SPPB total score remained significant $(B=-0.33, p<0.001)$ in the

Table 1. General characteristics of the study population

\begin{tabular}{|c|c|c|c|c|}
\hline & Total $(n=3,010)$ & $\operatorname{Men}(n=1,429)$ & Women $(n=1,581)$ & $\mathrm{p}$-value \\
\hline Age (y) & $76.5 \pm 3.9$ & $76.8 \pm 3.9$ & $76.2 \pm 3.9$ & $<0.001$ \\
\hline Education (y) & $8.7 \pm 6.3$ & $10.6 \pm 5.7$ & $6.7 \pm 6.2$ & $<0.001$ \\
\hline Nutritional status, MNA score & $12.8 \pm 1.5$ & $12.9 \pm 1.5$ & $12.8 \pm 1.5$ & 0.221 \\
\hline Cognitive function, MMSE score & $25.5 \pm 3.4$ & $26.1 \pm 3.0$ & $24.9 \pm 3.5$ & $<0.001$ \\
\hline Depressive status, GDS score & $3.3 \pm 3.7$ & $2.5 \pm 3.2$ & $4.0 \pm 4.0$ & $<0.001$ \\
\hline Body mass index $\left(\mathrm{kg} / \mathrm{m}^{2}\right)$ & $24.4 \pm 3.0$ & $23.9 \pm 2.9$ & $24.9 \pm 3.1$ & $<0.001$ \\
\hline Gait speed $(\mathrm{m} / \mathrm{s})$ & $1.1 \pm 0.3$ & $1.1 \pm 0.3$ & $1.1 \pm 0.2$ & $<0.001$ \\
\hline Grip strength $(\mathrm{kg})$ & $26.2 \pm 7.6$ & $32.1 \pm 6.0$ & $20.9 \pm 4.2$ & $<0.001$ \\
\hline Timed Up and Go test (s) & $10.6 \pm 2.9$ & $10.3 \pm 2.7$ & $10.8 \pm 3.0$ & $<0.001$ \\
\hline SPPB total score & $10.8 \pm 1.6$ & $11.1 \pm 1.3$ & $10.5 \pm 1.7$ & $<0.001$ \\
\hline Low SPPB (total score $\leq 9)$ & $549(18.2)$ & $175(12.3)$ & $374(23.7)$ & $<0.001$ \\
\hline CHS frailty criteria score ${ }^{a}$ & $1.1 \pm 1.8$ & $1.1 \pm 1.8$ & $1.2 \pm 1.8$ & 0.070 \\
\hline Robust & $1,325(45.6)$ & $696(50.4)$ & $629(41.3)$ & \\
\hline Prefrail & $1,362(46.9)$ & $591(42.8)$ & $771(50.6)$ & \\
\hline Frail & $218(7.5)$ & $95(6.9)$ & $123(8.1)$ & \\
\hline
\end{tabular}

Values are presented as mean \pm standard deviation or number (\%).

CHS, Cardiovascular Health Study; GDS, Geriatric Depression Scale; MMSE, Mini-Mental State Examination; MNA, Mini Nutritional Assessment Short Form; SPPB, Short Physical Performance Battery.

a) Analysis of CHS frailty scale in 1,382 men and 1,523 women. 

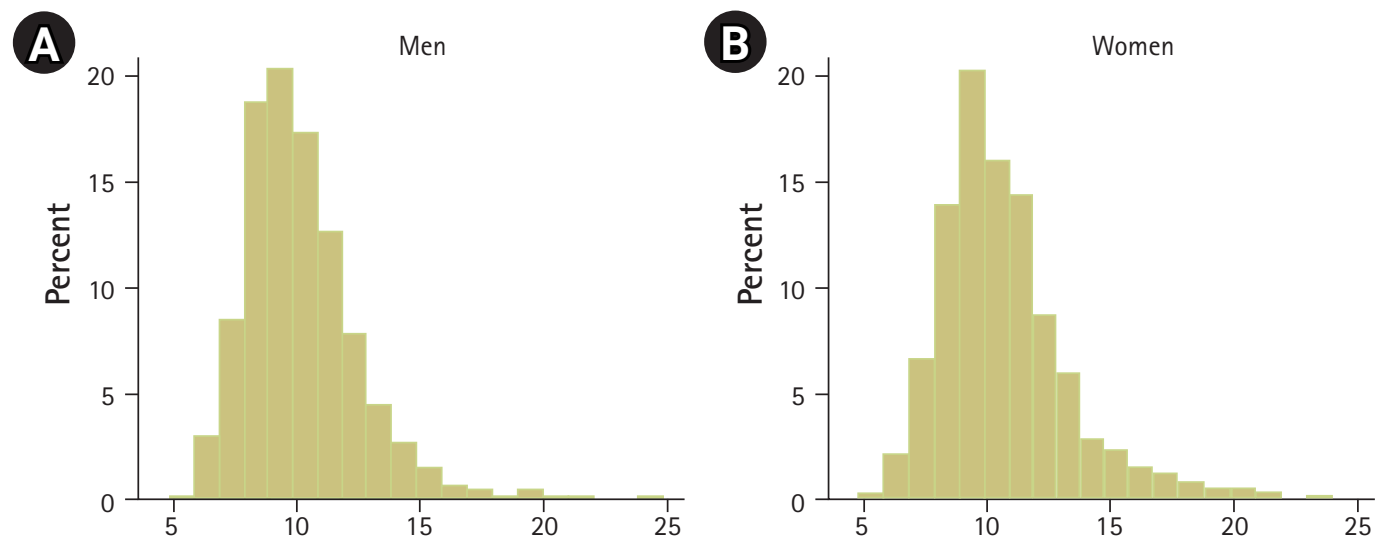

TUG time (s)*

\begin{tabular}{|l|c|c|c|c|c|c|c|c|c|c|c|c|c|c|}
\hline & \multicolumn{1}{|c|}{ Men } & \multicolumn{1}{c|}{ Women } \\
\hline Percentile (\%) & 20 & 25 & 40 & 50 & 60 & 75 & 80 & 20 & 25 & 40 & 50 & 60 & 75 & 80 \\
\hline Centile (s) & 8.3 & 8.6 & 9.3 & 9.8 & 10.3 & 11.3 & 11.8 & 8.6 & 9.0 & 9.7 & 10.2 & 10.9 & 12.0 & 12.5 \\
\hline
\end{tabular}

*In the graph, 5 men and 7 women with TUG time of $>25$ seconds are omitted.

Fig. 1. Distribution of Timed Up and Go test (TUG) time in men (A) and women (B).
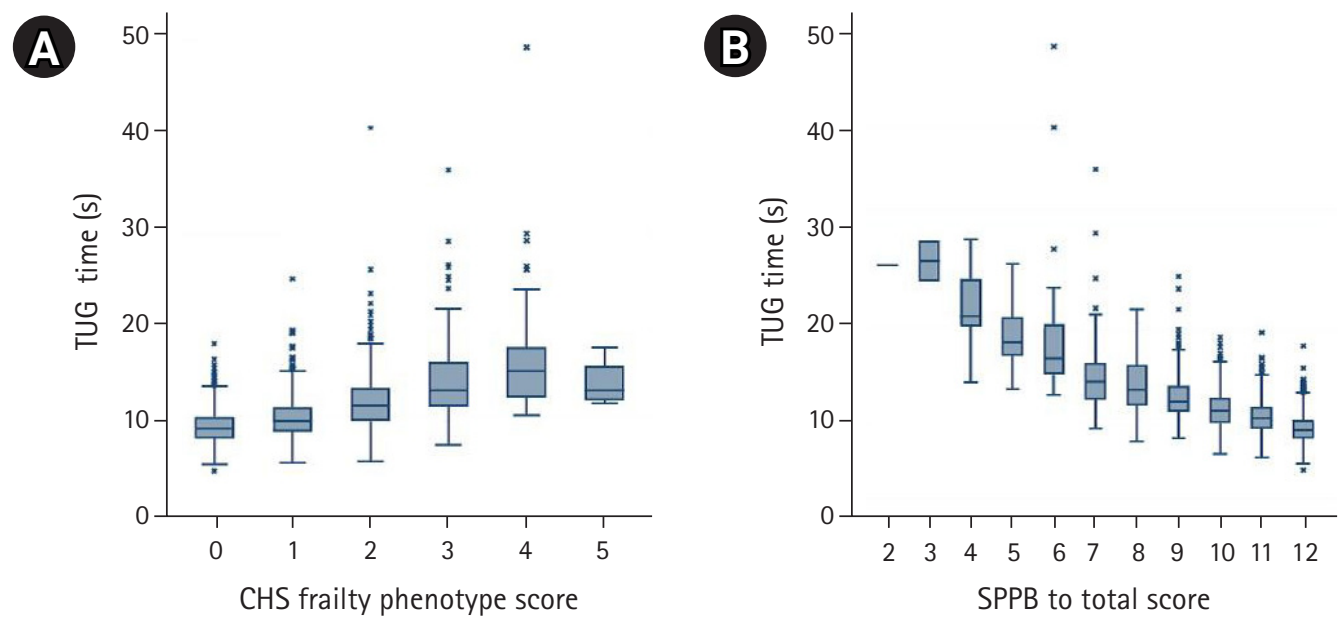

Fig. 2. Box plots showing distributions of Timed Up and Go test (TUG) time by the Cardiovascular Health Study (CHS) frailty phenotype score (A) and Short Physical Performance Battery (SPPB) total score.

multivariate linear regression analysis that included age and sex. Fig. 2 shows the corresponding TUG time distribution according to the CHS frailty scale score and SPPB total score. To assess content validity, correlations between TUG time and component-specific and total scores of the CHS frailty scale and SPPB were calculated using linear regression analysis, as shown in Table 2.

\section{Criterion Validity of TUG in Detecting Low Physical Performance and Frailty}

In the ROC analysis, the performance of TUG in identifying physical frailty, calculated as the area under the curve (AUC), was 0.87, while the AUC of TUG in identifying low physical performance according to SPPB was 0.86 (Fig. 3).

The calculated sensitivities and specificities of TUG time to classify physical frailty and low physical performance in men and women are displayed in Table 3. Based on the worst quintile TUG time cut-off of $\geq 11.8$ seconds for men and $\geq 12.5$ seconds for women, the sensitivity and specificity were $74.74 \%$ and $83.92 \%$, respectively, in men and $65.04 \%$ and $85.07 \%$, respectively, in women for frailty and $68.57 \%$ and $86.04 \%$, respectively, in men and $55.08 \%$ and $90.56 \%$, respectively, in women for low physical performance. 
Table 2. Correlations between TUG times with component-specific CHS frailty criteria and SPPB total scores

\begin{tabular}{|c|c|c|c|c|}
\hline & & & & \\
\hline & B & p-value & B & p-value \\
\hline CHS total score & 0.327 & $<0.001$ & 0.436 & $<0.001$ \\
\hline Unintended weight loss & 0.038 & 0.150 & 0.184 & $<0.001$ \\
\hline Poor grip strength & 0.288 & $<0.001$ & 0.310 & $<0.001$ \\
\hline Exhaustion & 0.131 & $<0.001$ & 0.232 & $<0.001$ \\
\hline Slow walking speed & 0.470 & $<0.001$ & 0.533 & $<0.001$ \\
\hline Low physical activity & 0.129 & $<0.001$ & 0.163 & $<0.001$ \\
\hline SPPB total score & -0.643 & $<0.001$ & -0.678 & $<0.001$ \\
\hline SPPB balance score & -0.403 & $<0.001$ & -0.362 & $<0.001$ \\
\hline SPPB walking speed score & -0.580 & $<0.001$ & -0.647 & $<0.001$ \\
\hline SPPB chair rise test score & -0.443 & $<0.001$ & -0.502 & $<0.001$ \\
\hline
\end{tabular}

B, standardized regression coefficient; TUG, Timed Up and Go test; CHS, Cardiovascular Health Study; SPPB, Short Physical Performance Battery.

a) Analysis of CHS frailty score in 1,382 men and 1,523 women.
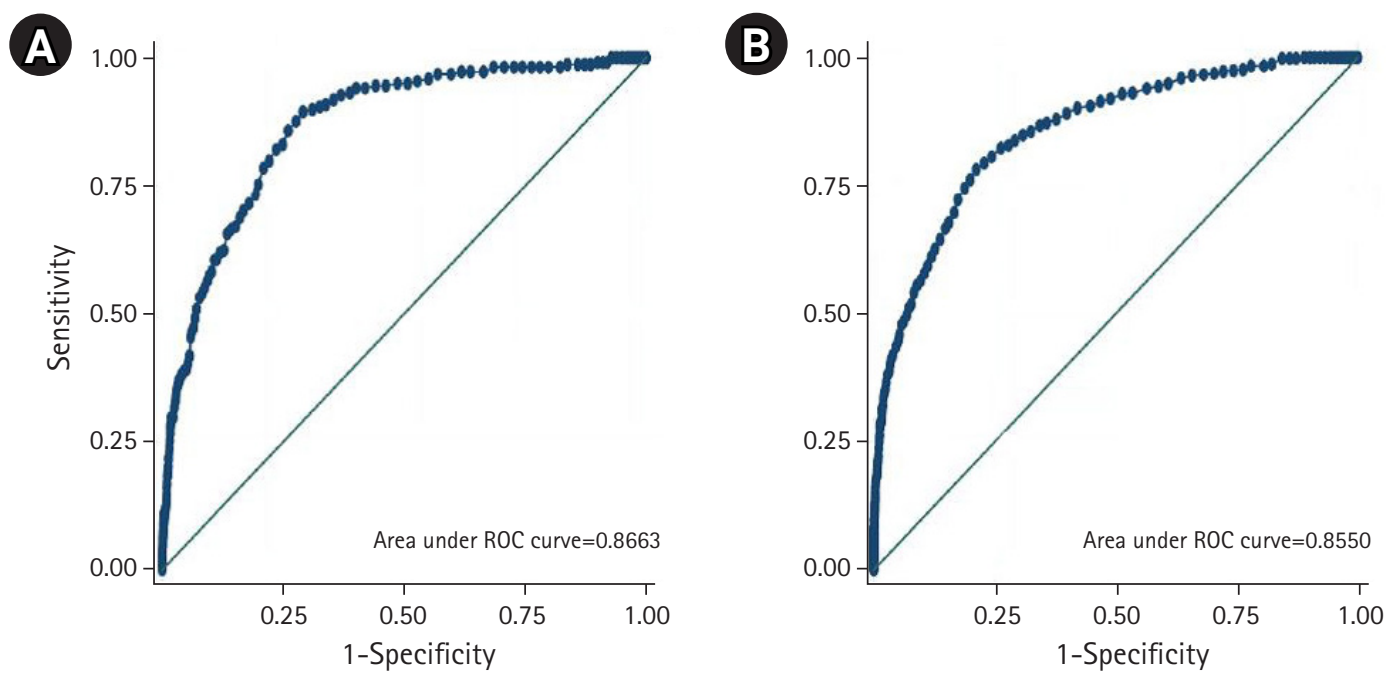

Fig. 3. Receiver operating characteristic (ROC) graphs of Timed Up and Go test time to classify frailty by the Cardiovascular Health Study criteria (A) and low physical performance by Short Physical Performance Battery (B).

\section{DISCUSSION}

In this study that used a nationwide population-based sample of Korean older adults, we found that TUG time correlated with component-specific and total scores of two widely accepted measures for physical frailty and physical performance. Our analysis revealed the criterion validity of TUG as a screening tool to classify physical frailty and low physical performance. In this study, the prevalence of low SPPB in men and women were $12.3 \%$ and $23.7 \%$, respectively, while the prevalence of $\mathrm{CHS}$ frailty were $6.9 \%$ and $8.1 \%$, respectively. In contrast, the prevalence of CHS frailty among men and women were $10.8 \%$ and $22.4 \%$, respectively, in the Aging Study of PyeongChang Rural Area (ASPRA) study and $3.5 \%$ and $16.5 \%$, respectively, in the Korean Longitudinal Study on Health and Aging (KLoSHA) study. ${ }^{22)}$ However, the previous studies had limitations because they were conducted only in limited rural or urban areas. ${ }^{22)}$ With KFACS as the first Korean multicenter prospective cohort on frailty, the significance of the present study is that it uses the nationwide distributions of frailty and low SPPB to produce corresponding TUG cut points. ${ }^{17)}$

Although many validated screening questionnaires exist for frail$t y{ }^{23-25)}$ subjective factors such as examiners' skill or perception and patients' prejudice regarding their health status may influence the results of these tools as these are measured using self-reported questionnaires. Alternatively, more objective and multifaceted assessments using the components of comprehensive geriatric assessment (CGA), which is considered to be the criterion standard for evaluating frailty, sometimes require more than 30 minutes to complete. $^{26,27)}$ Moreover, these in-depth methods have limitations 
Table 3. Sensitivity and specificity of TUG times to classify frailty according to the CHS criteria and low physical performance by SPPB

\begin{tabular}{|c|c|c|c|c|c|c|c|c|}
\hline \multirow{3}{*}{ TUG time $(\mathrm{s})$} & \multicolumn{4}{|c|}{ Frailty according to CHS phenotype } & \multicolumn{4}{|c|}{ Low physical performance according to SPPB } \\
\hline & \multicolumn{2}{|c|}{$\operatorname{Men}(n=1,382)$} & \multicolumn{2}{|c|}{ Women $(n=1,523)$} & \multicolumn{2}{|c|}{$\operatorname{Men}(n=1,429)$} & \multicolumn{2}{|c|}{ Women $(n=1,581)$} \\
\hline & Sensitivity (\%) & Specificity (\%) & Sensitivity (\%) & Specificity (\%) & Sensitivity (\%) & Specificity (\%) & Sensitivity (\%) & Specificity (\%) \\
\hline$\geq 8$ & 98.95 & 13.91 & 98.37 & 11.57 & 99.43 & 14.51 & 100.00 & 13.59 \\
\hline$\geq 9$ & 97.89 & 35.98 & 98.37 & 27.50 & 97.71 & 37.32 & 95.99 & 31.07 \\
\hline$\geq 10$ & 94.74 & 57.42 & 94.31 & 50.50 & 92.57 & 59.49 & 89.04 & 56.42 \\
\hline$\geq 11$ & 89.47 & 75.14 & 89.43 & 67.07 & 83.43 & 77.43 & 79.41 & 73.82 \\
\hline$\geq 12$ & 70.53 & 86.25 & 69.92 & 80.36 & 63.43 & 88.20 & 62.30 & 86.50 \\
\hline$\geq 13$ & 51.58 & 92.31 & 61.79 & 88.64 & 50.29 & 94.50 & 47.59 & 93.21 \\
\hline$\geq 14$ & 32.63 & 95.57 & 48.78 & 93.29 & 37.14 & 97.69 & 36.36 & 97.10 \\
\hline$\geq 15$ & 26.32 & 97.51 & 43.90 & 95.93 & 26.29 & 98.80 & 29.41 & 98.59 \\
\hline$\geq 16$ & 20.00 & 98.91 & 34.96 & 97.64 & 16.57 & 99.36 & 21.39 & 99.17 \\
\hline
\end{tabular}

TUG, Timed Up and Go test; CHS, Cardiovascular Health Study; SPPB, Short Physical Performance Battery.

that require dedicated personnel and space, precluding their widespread adoption in clinical practices.

The results showing the correlations between TUG time and components of CHS frailty criteria and SPPB were expected because TUG comprises elements of walking, chair rising, and sitting. Consequently, in correlation analyses, the coefficients were the highest for TUG time with the chair rise test of the SPPB and the walking speed item in the CHS frailty criteria. However, TUG time was also correlated with self-reported items such as exhaustion and low physical activity, suggesting that TUG reflects multiple facets of human health. Based on this observation, TUG, which can be performed quickly in spaces as small as 3-m long, might be both a suitable and feasible tool as an objective functional measure with minimal requirements for resources compared with 4-m or longer space required for gait speed measurement.

Using TUG as a primary screening tool for mobility and frailty has been advocated by societies and backed by clinical experiences and scientific evidence. ${ }^{9)}$ For example, the clinical practice guidelines of the American Geriatrics Society/British Geriatrics Society recommend using TUG as a primary physical functional assessment measure for fall risk. ${ }^{28)}$ In addition to the present study showing the construct validity and criterion validity of TUG for frailty and low physical performance, previous studies support the outcome validity of TUG in the Korean population for varying conditions associated with aging ${ }^{7,10-13)}$ in the Korean National Health Insurance Service-National Health Screening Cohort database, suggesting TUG as a valid screening tool in the Korean population.

Meanwhile, the cut-off value of TUG time has not yet been clearly defined, and TUG results depend on specific protocols (gait speed with usual or maximal effort) and various demographic backgrounds. In previous studies, $\geq 10$ seconds was mainly used as the cut-off value for TUG for fall risk and frailty, ${ }^{6,11-13)}$ When the
Youden index was applied in our data (Table 3), $\geq 11$ seconds appeared to be a preferred cut-off value for TUG in screening frailty. In contrast, when setting the cut-off time for the frailty index of deficit accumulation, ${ }^{29}$ ) the worst quintile ( $\geq 11.8$ seconds for men and $\geq 12.5$ seconds for women) might be used in the Korean population. However, since cut-off values can be modified and adapted for specific clinical and research environments, we did not provide a universal cut-off value in this study.

Our study had some limitations. As this was a cross-sectional analysis of a prospective cohort, it lacked outcome measures to provide the clinical outcome relevance of TUG in the study population; thus, future research with appropriate outcome data is needed. TUG in the KFACS was performed in relatively less constrained spaces in most centers, in contrast to many real-world outpatient clinics with spatially restricted environments, which may restrict the generalizability of our findings in real-world settings. In addition, according to the study design, the participants had to visit study centers, while people with severe frailty or dysmobility may have been less represented in the study population of the KFACS. Therefore, the distributions of TUG in this study population should be cautiously interpreted for older patients in clinical practice.

Nevertheless, the major strength of this study is that the sample size was relatively large and representative of the Korean population of community-dwelling ambulatory older adults aged between 70 and 84 years. Using this representative population, this study is, to our knowledge, the first to show the distribution of TUG time in the Korean population and the first to identify a cutoff TUG time as a screening tool for frailty using the original criteria of weakness and slowness of Fried frailty phenotype, which defines weakness as the lowest $20 \%$ at baseline adjusted for sex and BMI and slowness as the slowest $20 \%$ of the population adjusted 
for sex and standing height in the designated population.

In conclusion, TUG is a simple and valid screening tool for frailty and low physical performance in community-dwelling older adults in Korea.

\section{ACKNOWLEDGMENTS}

\section{CONFLICT OF INTEREST}

Hee-Won Jung cofounded Dyphi Inc. The other researchers claim no conflicts of interest.

\section{FUNDING}

This study was funded by the Ministry of Health \& Welfare, Republic of Korea (No. HI15C3153). This study was also supported by a grant from the Korea Health Technology R\&D Project through the Korean Health Industry Development Institute (KHIDI) and by the Korean Geriatrics Society Research Challenge Award (2019).

\section{AUTHOR CONTRIBUTIONS}

Conceptualization, HWJ, SK; Data curation, HWJ, SK; Funding acquisition, CWW; Investigation, HWJ, SK; Methodology, HWJ, SK; Writing-original draft, HWJ, SK; Writing-review \& editing, all.

\section{SUPPLEMENTARY MATERIALS}

Supplementary materials can be found via http://doi.org/10.4235/ agmr.20.0072

\section{REFERENCES}

1. Jung HW. Visualizing domains of comprehensive geriatric assessments to grasp frailty spectrum in older adults with a radar chart. Ann Geriatr Med Res 2020;24:55-6.

2. Hoogendijk EO, Afilalo J, Ensrud KE, Kowal P, Onder G, Fried LP. Frailty: implications for clinical practice and public health. Lancet 2019;394:1365-75.

3. Clegg A, Young J, Iliffe S, Rikkert MO, Rockwood K. Frailty in elderly people. Lancet 2013;381:752-62.

4. Fried LP, Tangen CM, Walston J, Newman AB, Hirsch C, Gottdiener J, et al. Frailty in older adults: evidence for a phenotype. J Gerontol A Biol Sci Med Sci 2001;56:M146-56.

5. Cesari M. Physical frailty and sarcopenia: development of a framework for supporting interventions against incident mobility disability. Ann Geriatr Med Res 2017;21:42-8.

6. Podsiadlo D, Richardson S. The timed "Up \& Go": a test of basic functional mobility for frail elderly persons. J Am Geriatr Soc
1991;39:142-8.

7. Viccaro LJ, Perera S, Studenski SA. Is timed up and go better than gait speed in predicting health, function, and falls in older adults? J Am Geriatr Soc 2011;59:887-92.

8. Savva GM, Donoghue OA, Horgan F, O'Regan C, Cronin H, Kenny RA. Using timed up-and-go to identify frail members of the older population. J Gerontol A Biol Sci Med Sci 2013;68: 441-6.

9. Clegg A, Rogers L, Young J. Diagnostic test accuracy of simple instruments for identifying frailty in community-dwelling older people: a systematic review. Age Ageing 2015;44:148-52.

10. Yoo JE, Jang W, Shin DW, Jeong SM, Jung HW, Youn J, et al. Timed Up and Go Test and the risk of Parkinson's disease: a nation-wide retrospective cohort study. Mov Disord 2020;35: 1263-7.

11. Lee JE, Chun H, Kim YS, Jung HW, Jang IY, Cha HM, et al. Association between Timed Up and Go Test and subsequent functional dependency.J Korean Med Sci 2020;35:e25.

12. Chun S, Shin DW, Han K, Jung JH, Kim B, Jung HW, et al. The Timed Up and Go test and the ageing heart: Findings from a national health screening of 1,084,875 community-dwelling older adults. Eur J Prev Cardiol 2019 Oct 20 [Epub]. https://doi. org/10.1177/2047487319882118.

13. Jeong SM, Shin DW, Han K, Jung JH, Chun S, Jung HW, et al. Timed up-and-go test is a useful predictor of fracture incidence. Bone 2019;127:474-81.

14. Won CW, Lee Y, Choi J, Kim KW, Park Y, Park H, et al. Starting construction of frailty cohort for elderly and intervention study. Ann Geriatr Med Res 2016;20:114-7.

15. Guralnik JM, Ferrucci L, Pieper CF, Leveille SG, Markides KS, Ostir GV, et al. Lower extremity function and subsequent disability: consistency across studies, predictive models, and value of gait speed alone compared with the short physical performance battery. J Gerontol A Biol Sci Med Sci 2000;55:M221-31.

16. Bauer JM, Verlaan S, Bautmans I, Brandt K, Donini LM, Maggio $\mathrm{M}$, et al. Effects of a vitamin $\mathrm{D}$ and leucine-enriched whey protein nutritional supplement on measures of sarcopenia in older adults, the PROVIDE study: a randomized, double-blind, placebo-controlled trial. J Am Med Dir Assoc 2015;16:740-7.

17. Won CW, Lee S, Kim J, Chon D, Kim S, Kim CO, et al. Korean frailty and aging cohort study (KFACS): cohort profile. BMJ Open 2020;10:e035573.

18. Orme JG, Reis J, Herz EJ. Factorial and discriminant validity of the Center for Epidemiological Studies Depression (CES-D) scale. J Clin Psychol 1986;42:28-33.

19. Kang Y, Na DL, Hahn S. A validity study on the Korean Mini-Mental State Examination (K-MMSE) in dementia pa- 
tients. J Korean Neurol Assoc 1997; 15:300-8.

20. Jung IK, Kwak DI, Joe SH, Lee HS. A study of standardization of Korean form of Geriatric Depression Scale (KGDS). J Korean Geriatr Psychiatry 1997;1:61-72.

21. Vellas B, Guigoz Y, Garry PJ, Nourhashemi F, Bennahum D, Lauque S, et al. The Mini Nutritional Assessment (MNA) and its use in grading the nutritional state of elderly patients. Nutrition 1999; 15:116-22.

22. Jang IY, Jung HW, Lee CK, Lee YS, Kim KI, Kim KW, et al. Rural and urban disparities in frailty and aging-related health conditions in Korea. J Am Geriatr Soc 2016;64:908-11.

23. Kim S, Kim M, Jung HW, Won CW. Development of a frailty phenotype questionnaire for use in screening community-dwelling older adults. J Am Med Dir Assoc 2020;21:660-4.

24. Ambagtsheer RC, Archibald MM, Lawless M, Mills D, Yu S, Beilby JJ. General practitioners' perceptions, attitudes and experiences of frailty and frailty screening. Aust J Gen Pract 2019;48: 426-33.

25. Jung HW, Yoo HJ, Park SY, Kim SW, Choi JY, Yoon SJ, et al. The
Korean version of the FRAIL scale: clinical feasibility and validity of assessing the frailty status of Korean elderly. Korean J Intern Med 2016;31:594-600.

26. Jung HW, Kang MG, Choi JY, Yoon SJ, Kim SW, Kim KI, et al. Simple method of screening for frailty in older adults using a chronometer and tape measure in clinic. J Am Geriatr Soc 2018; 66:157-60.

27. Dent E, Martin FC, Bergman H, Woo J, Romero-Ortuno R, Walston JD. Management of frailty: opportunities, challenges, and future directions. Lancet 2019;394:1376-86.

28. Panel on Prevention of Falls in Older Persons; American Geriatrics Society and British Geriatrics Society. Summary of the Updated American Geriatrics Society/British Geriatrics Society clinical practice guideline for prevention of falls in older persons. J Am Geriatr Soc 2011;59:148-57.

29. Searle SD, Mitnitski A, Gahbauer EA, Gill TM, Rockwood K. A standard procedure for creating a frailty index. BMC Geriatr 2008;8:24. 